\title{
A new role of lipid receptors in vascular and cardiac morphogenesis
}

\author{
Thomas N. Sato
}

The University of Texas Southwestern Medical Center at Dallas, 5323 Harry Hines Boulevard, NB11.106, Dallas, Texas 75390-8573, USA. Phone: (214) 648-1402; Fax: (214) 648-1433; E-mail: island1005@aol.com.

Diverse classes of molecules regulate cell migration, one of the most fundamental processes in development and disease. In the vascular system, smooth muscle cell migration mediated by the PDGF-B pathway (1) is required for both normal blood vessel formation during development and pathogenesis of vascular diseases. In this paracrine signaling pathway, endothelial cells secrete PDGF-B, which acts on the PDGF- $\beta$ receptor expressed on the surface of smooth muscle cell precursors and initiates the migration of the latter cells toward endothelial cells during angiogenesis.

In this issue of the JCI, Liu et al. implicate another receptor and another class of mediator - bioactive glycolipids - in smooth muscle cell migration in the mouse (2). Remarkably, this same class of molecule was recently shown to mediate the migration of cardiac muscle progenitor cells during the initial formation of the heart tube in zebrafish development (3). Together, these two studies establish the presence of a common pathway, mediated by receptors for sphingosine-1-phosphate (S1P), underlying both smooth muscle and cardiac muscle migration during vascular and cardiac development (Figure 1).

In the present study, Liu et al. (2) address the putative biological functions of one of the S1P receptors, Edg1 , by generating knockout mice for its gene. Edg-1 belongs to a family of $\mathrm{G}$ protein-coupled receptors for S1P or lysophosphatidic acid $(4,5)$. Edg- 1 is a specific receptor for $\mathrm{S} 1 \mathrm{P}$ and interacts directly with the $G_{i}$ pathway. Targeted mutation of Edg-1 resulted in embryonic lethality (2), but the most intriguing feature of the Edg-1 knockout phenotype is the apparent lack of normal layers of smooth muscles surrounding endothelial cells of vessels (2). Interestingly, smooth muscle cells are clustered to the ventral side of the vessels and fail to surround the endothelial lumen of the vessels in a uniform fashion. Smooth muscle cell precursor cells have been thought to be recruited from any direction in the vessel's vicinity (1). However, this polarized clustering of smooth muscle cells in Edg1 mutant embryos suggests an alternative model in which smooth muscle cells are recruited in a unidirectional manner (6, 7 ) and only later spread to surround the endothelium. This unidirectional recruitment is not understood, but it might be explained by an asymmetrically distributed chemoattractant gradient or by the presence of a critical adhesive factor on the ventral side of the developing vessel.

In PDGF-B-deficient mice, in contrast, pericytes (contractile cells for capillaries equivalent to smooth muscle cells for larger vessels) are not unevenly distributed but are actually absent from vessels (8). This difference suggests the following model for the smooth muscle formation in developing vessels (Figure 2): PDGF-B, secreted by endothelial cells undergoing angiogenesis, mediates the recruitment of smooth muscle precursors toward the immediate vicinity of the endothelial cells, where
Figure 1

New roles of lipid receptors in cardiac and vascular development. Receptors for S1P are critical for the migration of cardiac muscle precursors and smooth muscle cells during heart tube formation and blood vessel formation, respectively. See the text for details. White boxes, Edg-1 (vessel), mil (heart); purple arrows, S1P; blue cells, endothelial cells (vessel), midline cells (heart); orange cells, smooth muscle cells (vessel), cardiac muscle precursors; green arrows, chemoattractant(s) or permissive microenvironment. they accumulate in an asymmetric fashion. Once the smooth muscle precursors are immediately adjacent to endothelial cells, they are induced to differentiate to mature smooth muscle cells via signaling pathways including TGF- $\beta$, endoglin, and SMADs (1, $9-11)$. Following the formation of a complete vascular lumen by endothelial cells, S1P mediates the migration of smooth muscle cells either directly or indirectly to surround the endothelial layer. Because smooth muscle cell migration goes awry in many types of vascular disease $(12,13)$, this model could have profound clinical as well as biological implications. It is conceivable that the same or a related lipid receptor signaling pathway is critically involved in or could be exploited to treat human diseases.

\section{Instructive parallels}

Recently published zebrafish work on another S1P receptor, mil (mile apart), clearly shows the existence of a parallel pathway between cardiac and smooth muscle cell migration (Figure 1). Mil belongs to the same class of receptors as Edg-1 and is most closely related to Edg-5 (3). As shown for Edg-5, S1P induces both intracellular calcium mobilization and mitogen-activated protein kinase activation in mil-transfected cells (3). In the mil mutant zebrafish, cardiac muscle precursors fail to migrate toward the midline, an

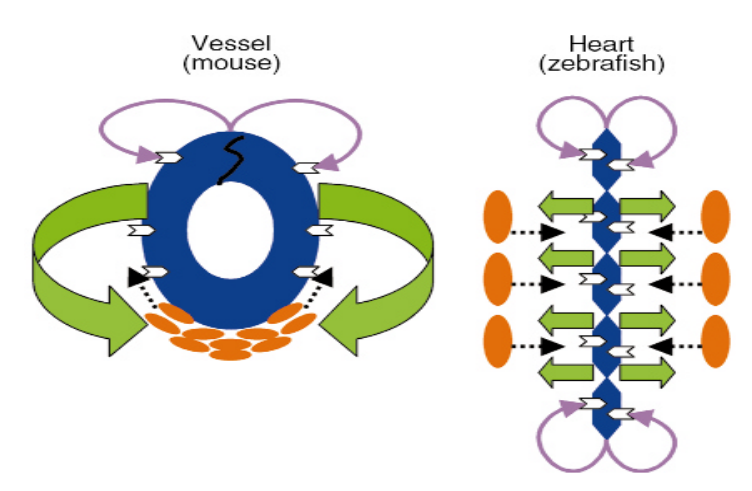




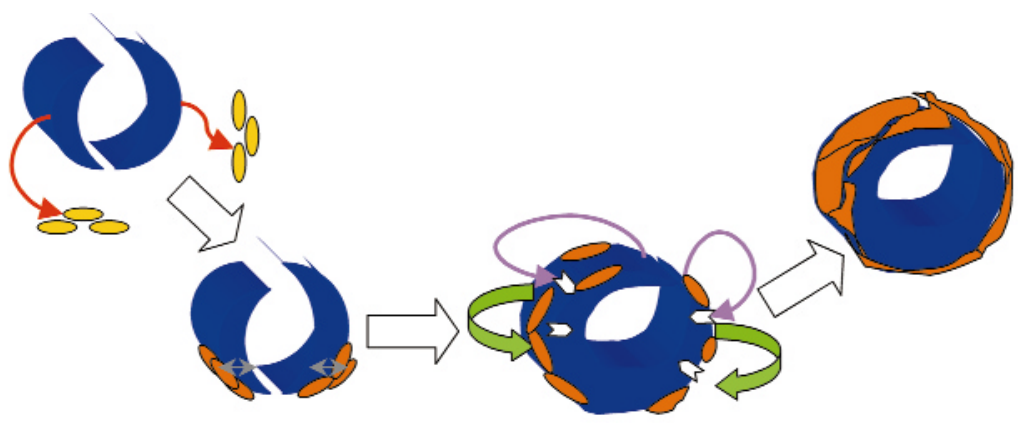

Figure 2

Sequential roles of PDGF-B/TGF- $\beta / S 1 \mathrm{P}$ in smooth muscle infiltration into vessels. During angiogenesis, endothelial cells that are yet to form a mature vascular lumen secrete PDGF-B to recruit smooth muscle precursor cells to their immediate vicinity. Once the smooth muscle precursors are recruited, they are induced to differentiate to smooth muscle cells via activation of TGF- $\beta$ pathways. As endothelial cells form a new lumen during angiogenesis, differentiated smooth muscle cells migrate along the endothelial cells to surround the vessels and establish a mature vasculature. This later process seems to be regulated via S1P. Two possible mechanisms are proposed for the action of S1P. S1P may control smooth muscle cell migration directly. Alternatively, S1P may act on other cells such as endothelial cells and indirectly regulates the migration of smooth muscle cells, either by inducing the expression of a putative chemoattractant(s) or by generating a permissive microenvironment for the migration. Work in a parallel developmental pathway in zebrafish argues for the latter model. Yellow cells, smooth muscle precursors; dark orange arrows, PDGF-B; blue cells, endothelial cells; orange cells, smooth muscle cells; gray arrows, TGF- $\beta$; white boxes, Edg-1; purple arrows, S1P; green arrows, chemoattractant(s) or permissive microenvironment.

initial process of cardiac tube formation during development (3). Interestingly, mil function is not required in migrating cardiac muscle precursors, since mil mutant cells migrate normally when transplanted in the wild-type embryo. However, when wild-type cells are transplanted into the mil mutant embryos, they fail to migrate normally. Furthermore, mil expression is detected lateral to the midline during initial heart tube formation, suggesting that $\mathrm{S} 1 \mathrm{P}$ activates mil in cells located at the midline. This S1P-mediated mil activation, in turn, induces the expression of secondary chemoattractants or induces a permissive microenvironment for the migration of cardiac muscle precursors toward the midline in order to form the cardiac tube.

These analyses of Edg1 in mice and mil in zebrafish establish an important role of lipid receptor-mediated signaling pathways in vascular and cardiac development. Clearly, however, they leave a number of outstanding biological and medical questions to be addressed in the near future. First, the metabolic pathways that regulate S1P function must be clarified. S1P activity is modulated by kinases and phosphatases, but the regulation and location of S1P biosynthesis and

degradation are not well understood. Second, the downstream targets of S1P that directly regulate muscle cell migration remain to be defined. In particular, the cell types are not known that depend on Edg-1 function to activate smooth muscle cell migration. In zebrafish, mil function is not required in the migrating cardiac muscle precursors, suggesting that S1P generates a permissive microenvironment for the migration of muscle precursors or cells, perhaps by inducing the secretion of paracrine chemoattractants (Figure 1). By analogy, it is possible that S1P acts on endothelial cells during angiogenesis and that these endothelial cells secrete chemoattractants for smooth muscle cell migration. Future studies using cell type-specific knockouts and cell type-specific expression of Edg-1 to rescue the knockout phenotype will be useful in addressing this question. Finally, the physiological role of S1P and other related compounds needs to be established. The present findings (2) show that S1P interacts with Edg-1 in cell culture, but they do not exclude the possibility that other lipids or glycolipids serve as natural ligands for Edg- 1 and mil and activate cell migration in vivo.
The parallels between the Edg-1 and mil pathways hint at a previously unsuspected principle unifying angiogenesis and cardiac morphogenesis as they occur in disparate vertebrate species. Fortunately, the conservation of this fundamental pathway - which may extend still further across the phylogenic tree should allow for progress on these questions to occur on many fronts at once, taking advantage of mouse and zebrafish genetics, as well as other appropriate systems for developmental studies.

\section{Acknowledgments}

The author would like to acknowledge Siobhan Loughna, Richard Visconti, and Charlene Richardson for critical comments on this Commentary. The author would also like to thank John Ashkenas for editing and discussion during its preparation. Thomas N. Sato is an Established Investigator of the American Heart Association.

1. Folkman, J., and D’Amore, P.A. 1996. Blood vessel formation: What is its molecular basis? Cell. 87:1153-1155.

2. Liu, Y., et al. 2000. Edg-1, the G protein-coupled receptor for sphingosine-1-phosphate, is essential for vascular maturation. J. Clin. Invest. 106:951-961

3. Kupperman, E., An, S., Osborne, N., Waldron, S., and Stainier, D.Y. 2000. A sphingosine-1-phosphate receptor regulates cell migration during vertebrate heart development. Nature. 406:192-195.

4. Hla, T., and Maciag, T. 1990. An abundant transcript induced in differentiating human endothelial cells encodes a polypeptide with structural similarities to G-protein-coupled receptors. J. Biol. Chem. 265:9308-9313.

5. Lee, M.J., et al. 1998. Sphingosine-1-phosphate as a ligand for the $G$ protein-coupled receptor EDG-1. Science. 279:1552-1555.

6. Hungerford, J.E., et al. 1997. Identification of a novel marker for primordial smooth muscle and its differential expression pattern in contractile vs noncontractile cells. J. Cell Biol. 137:925-937.

7. Lee, S.H., Hungerford, J.E., Little, C.D., and Iruela-Arispe, M.L. 1997. Proliferation and differentiation of smooth muscle cell precursors occurs simultaneously during the development of the vessel wall. Dev. Dyn. 209:342-352.

8. Lindahl, P., Johansson, B.R., Leveen, P., and Betsholtz, C. 1997. Pericyte loss and microaneurysm formation in PDGF-B-deficient mice. Science. 277:242-245.

9. Li, D.Y., et al. 1999. Defective angiogenesis in mice lacking endoglin. Science. 284:1534-1537.

10. Chang, H., et al. 1999. Smad5 knockout mice die at mid-gestation due to multiple embryonic and extraembryonic defects. Development. 126:1631-1642.

11. Yang, X., et al. 1999. Angiogenesis defects and mesenchymal apoptosis in mice lacking SMAD5. Development. 126:1571-1580.

12. Ross, R. 1993. The pathogenesis of atherosclerosis: a perspective for the 1990s. Nature. 362:801-809.

13. Vikkula, M., et al. 1996. Vascular dysmorphogenesis caused by an activating mutation in the receptor tyrosine kinase TIE2. Cell. 87:1181-1190. 